\title{
Successful medical treatment of west syndrome with a KCNA2 variant: a case report
}

\author{
Han Wang ${ }^{\dagger}$, Yanwei Zhu ${ }^{\dagger}$, Dezhi Cao* ${ }^{*}$, Hong Chen, Xin Ding, Qi Zeng, Huafang Zou and Jianxiang Liao
}

\begin{abstract}
Background: West syndrome is a devastating disorder characterized by a triad of epileptic spasms, abnormal electroencephalography (EEG), and developmental arrest or psychomotor delay. In addition to early diagnosis, knowing the etiology of the condition is also important for its treatment. Among various etiologies, the genetic factors, especially mutations of ion channel genes, are very common and strongly linked to West syndrome.

Case presentation: A boy who had epileptic spasms from the age of 4 months was diagnosed with West syndrome based on the clinical manifestation and EEG results in Shenzhen Children's Hospital in June 2019. Trios whole-exome sequencing (WES) test and protein structural model prediction were performed. We also reviewed the clinical and genetic features of this syndrome and the mechanisms of action of topiramate (TPM) by literature search in databases of Online Mendelian Inheritance in Man, Clinical Genome Resource, PubMed, Chinese National Knowledge Infrastructure and Wanfang database using keywords "KCNA2" "West syndrome" and "Topiramate" by December 2020. The relationship between the effect of TPM and the pathogenesis of the KCNA2 variant was also assessed. The WES test revealed c.244C > T/p. Arg82Cys varaint of KCNA2 (NM_004974.3) in this patient, and Sanger sequencing identified this was a de novo mutation. As far as we know, this is the first report of the c.244C > T/p. Arg82Cys variant in KCNA2, which was likely a pathogenic mutation. The seizures were successfully controlled for 10 months by TPM after failure of sodium valproate, large doses of vitamin B6, and adrenocorticotropic hormone. We speculate that the therapeutic effect of TPM in this patient is partially due to the inhibition of carbonic anhydrase.
\end{abstract}

Conclusions: Mutations in the KCNA2 gene should be considered for patients with West syndrome. The TPM treatment is probably effective for KCNA2-associated disorders.

Keywords: Epilepsy, Gene variation, KCNA2, West syndrome, Topiramate

\section{Background}

West syndrome is one of the most common epileptic encephalopathies in the first 2 years of life [1]. It is characterized by a triad of epileptic spasms, hypsarrhythmia on interictal EEG, and developmental arrest or psychomotor delay [2, 3]. The seizures of West syndrome are difficult to control. However, a small proportion of West syndrome cases has a good prognosis after optional

*Correspondence: caodezhi888@aliyun.com

tHan Wang and Yanwei Zhu contributed equally to this work. Neurology Department, Shenzhen Children's Hospital affiliated to China Medical University, Shenzhen 518038, China therapy, so it is important to determine the etiology of the condition. To date, West syndrome caused by KCNA2 gene variation has not been reported. Here, we report for the first time a case of West syndrome caused by the c.244C > T (p.Arg82Cys) pathogenic variant of KCNA2, which highlights the clinical and behavioral aspects of the syndrome. Meanwhile, we successfully controlled the seizures using TPM following adrenocorticotropic hormone therapy. The relationship between the KCNA2-related genetic cause and the mechanism of action of TPM was explored. This study advances our understanding of the genetic and phenotypic spectrum of KCNA2-associated West syndrome. 


\section{Case presentation}

A 6-month-old Chinese boy with a history of seizures for over 2 months was brought to Shenzhen Children's Hospital in June 2019. The onset of seizures was at the age of 4 months, with spasms occurring in clusters of head nodding, peculiar spells, and sudden flexion of the trunk and extension of the limbs, which lasted 2-3 min on average. The seizures occurred 3-5 times daily. The frequency of seizures increased gradually, and all of the events were stereotyped and usually occurred just after he woke up.

The boy was the first child of a healthy, geneticallyunrelated Chinese couple. He was born normally at fullterm following an uneventful vaginal delivery at weight of $3.2 \mathrm{~kg}$ (standard deviation score [SDS]: -0.31 SD). He had slight asphyxia at birth, and his mother had gestational diabetes. His early development was slightly delayed. By the age of 4 months, he could hold his head up but could not roll over. The pursuit of light and objects was slightly poor, as was his response to sound. Any possible history of inherited metabolic disease, encephalitis or traumatic brain injury was denied.

Physical examination showed a weight and head circumference of $6.0 \mathrm{~kg}(<-2 \mathrm{SD})$ and $43.0 \mathrm{~cm}(-0.25$ $\mathrm{SD})$, respectively. Abnormal hyperpigmentation was not noted. His head control and visual pursuit remained slightly poor. Eyeballs and pupils had normal size, shape, equality and light reflexes, eye movement was normal, and no nystagmus was noted. All of his extremities had normal muscle tone.

Laboratory tests showed normal results for routine blood test, biochemical tests, and urine and blood metabolic tests.

The interictal EEG at age 6 months (on June 20, 2019) showed diffuse high-amplitude slow waves especially in the bilateral posterior regions, and multifocal epileptiform discharges. During sleep, there were bursts of spikes and slow waves. Epileptic spasms with paroxysmal diffuse EEG, as well as prismatic electromyography on electromyography, were observed (Fig. 1). Brain magnetic resonance imaging (MRI) showed widened subarachnoid space and formation of arachnoid cysts in the right temporal lobe; no abnormalities were found following brain parenchyma MRI.

\section{Treatment}

The patient was treated with sodium valproate and large doses of vitamin B6. The seizures remained uncontrolled after this treatment. Adrenocorticotropic hormone $(1-2 \mathrm{U} / \mathrm{kg})$ was injected intramuscularly twice daily, which slightly lowered the seizure frequency. However, the patient still experienced 15-25 single spasms a day after the 2-week therapy. Ten days later, TPM was added on at a gradually increased dose. The seizures were completely controlled after the TPM $(2 \mathrm{mg} / \mathrm{kg})$ administration.

We recorded EEGs from 6 months to 1 year and 3 months (from June 2019 to March 2020). We found that the epileptic seizures were gradually controlled, and follow-up EEG returned to near normal and was maintained steady (Figs. 1, 2 and 3). At the age of 1 year and 5 months, the seizures were steadily controlled for 10 months. The boy was able to walk alone, climb up and out of bed on his own, call "Mama" and "Papa" with a smile, respond to name calling and learn to talk, and had grown 4 teeth, similar to other normal children at the same age.

\section{Cytogenetic and molecular studies}

Blood genomic DNA was routinely extracted for exon group capture and sequencing of the exome. WES revealed presence of the c. $244 \mathrm{C}>\mathrm{T} / \mathrm{p}$. Arg82Cys heterozygous variant in the KCNA2 gene (NM_004974.3) in this patient. However, Sanger sequencing of parental DNA revealed no variation in this locus. This suggested that the c. $244 \mathrm{C}>\mathrm{T} / \mathrm{p}$. Arg82Cys mutation in the KCNA2 gene was a de novo mutation, a first case report so far. The carrying-rate of this variant in normal populations recorded in the gnomAD database is $0.4 \times 10^{-5}$. This variant is associated with the early-onset infantile epileptic encephalopathy type 32, and is inherited in an autosomal dominant manner. According to the guidelines of the American College of Medical Genetics and Genomics in 2015 [4], it is likely a pathogenic mutation (Fig. 4). No other pathogenic variants were present in the exome data. In addition, the $3 \mathrm{D}$ protein structure model was predicted by the Mutalyzer website and SWISS-MODEL (Fig. 5).

\section{Discussion}

In this report, we reported a 6-month-old boy who presented with epileptic spasms from the age of 4 months, hypsarrhythmia on interictal EEG, and psychomotor delay in development. Common blood counts, biochemical tests, and urine and blood metabolic investigations were all normal. Brain MRI showed no obvious abnormality. WES revealed presence of the c. $244 \mathrm{C}>\mathrm{T} / \mathrm{p}$.Arg82Cys variant of the KCNA2 gene (NM_004974.3), which is inherited in an autosomal dominant manner and is likely pathogenic. Finally, we successfully controlled the seizures by TPM therapy alone, and the results of EEG and clinical follow-up showed a good prognosis.

The KCNA2 gene is located on chromosome 1p13.3, a member of the voltage-gated potassium channel Kv1 family, encodes the voltage-gated delayed rectifier potassium channel $\alpha$-subunit Kv1.2 [5], which is abundantly expressed in the large axon terminals of basket cells of the central nervous system (CNS) that make powerful 


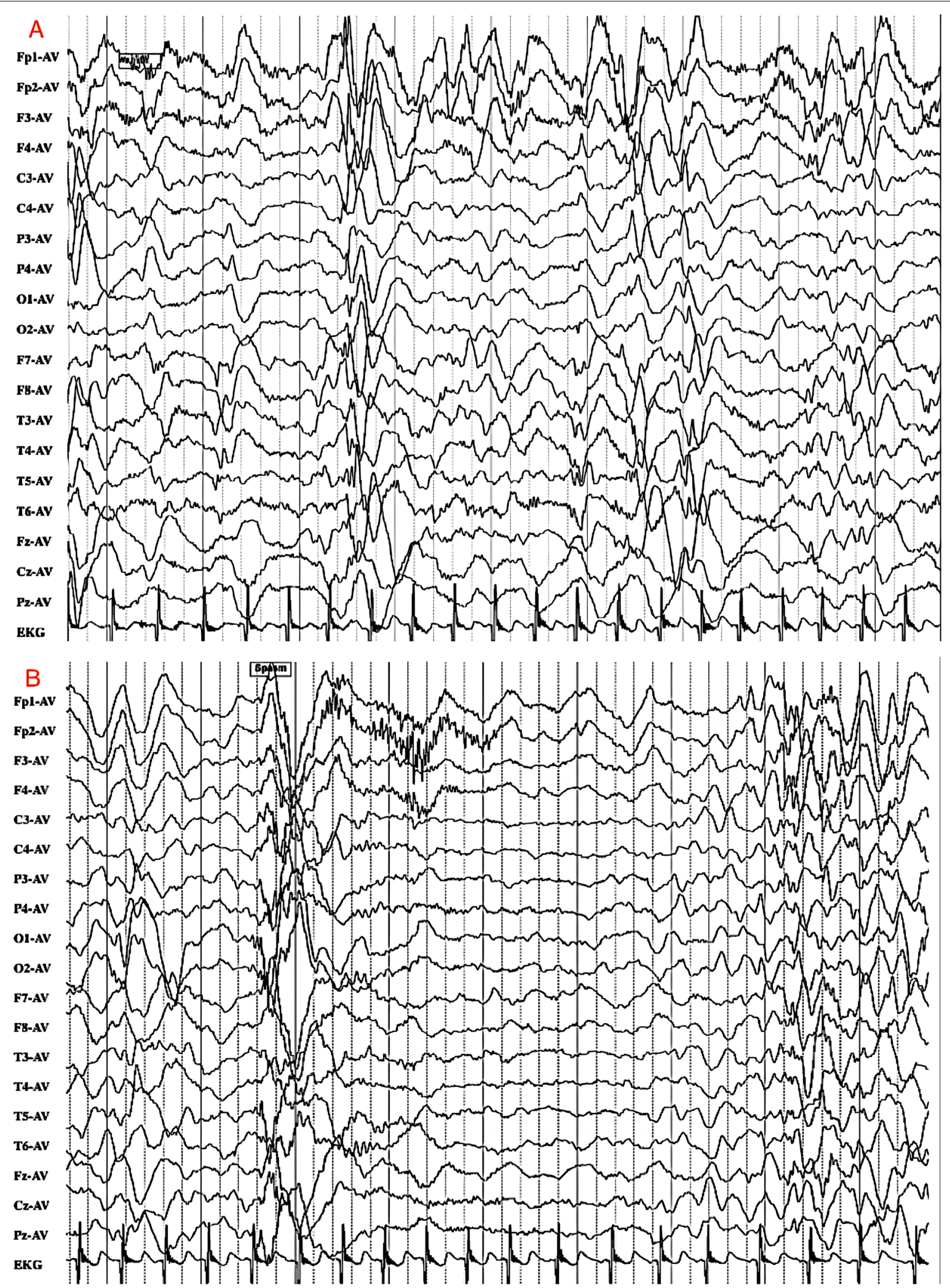

Fig. 1 Abnormal EEG at age of 6 months (on June 20, 2019). The background activity was completely disorganized and chaotic on interictal EEG, showing hypsarrhythmia. When the patient was awake, the susceptible ictal EEG showed flexor spasms 
A

\section{Ipl-AV}

Tp2-AV

F3-AV

J4-AV

CJ-AV

C4-AV

133.AV

P4-AV

O1-AV

02.AV

17.AV

WWAV

T3-AV

TA-AV

T5-AV

Tr.AV

F2-AV

Cz-AV

$\mathbf{P}$ waN

EKG

B

Fpl-AV

FP2-AV

F3-AV

F4-AV

C3-AV

CA-AV

PB-AV

PA-AV

O1-AV

02-AV

17.AV

FH-AV

T3-AV

THAN

TS-AV

'T6. AV

$\mathbf{H z}-\mathbf{A} \mathbf{V}$

CreAv

PzoAV

BKC;

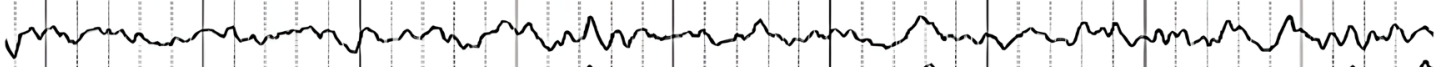

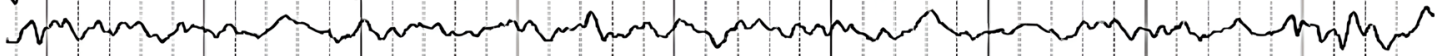

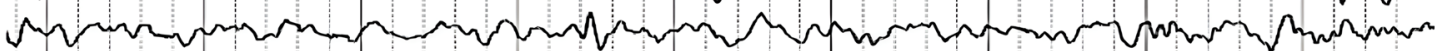

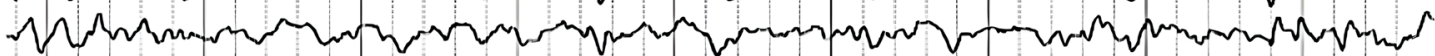

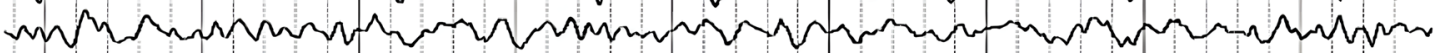

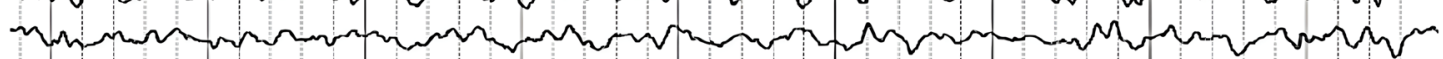

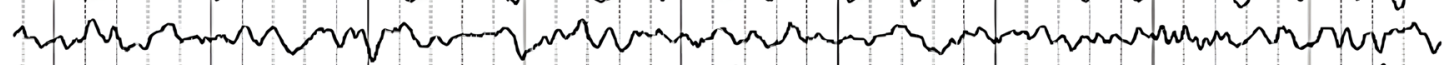

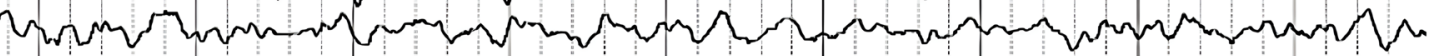

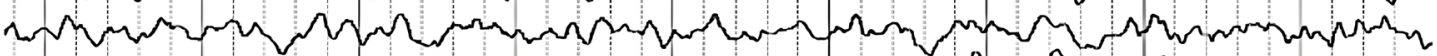

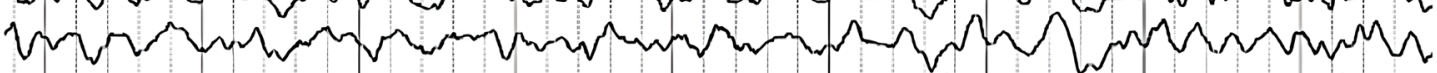
(n)

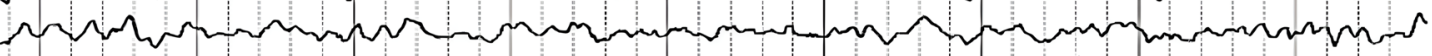

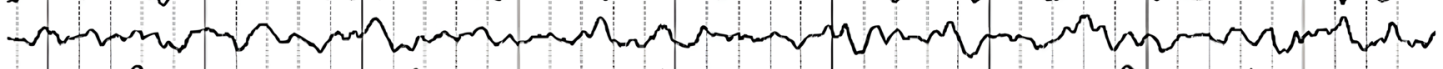

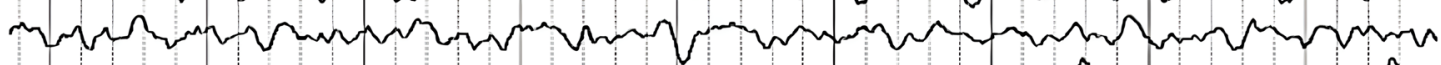

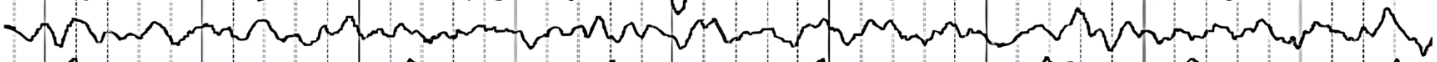

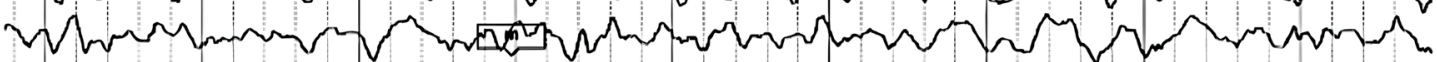

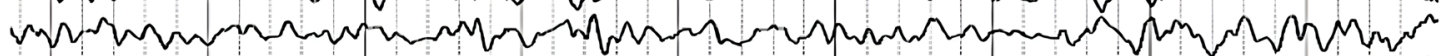

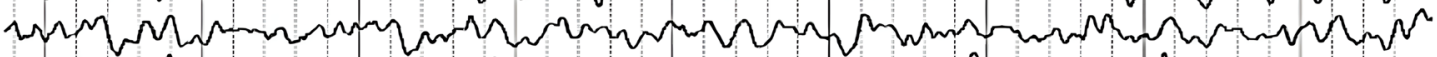

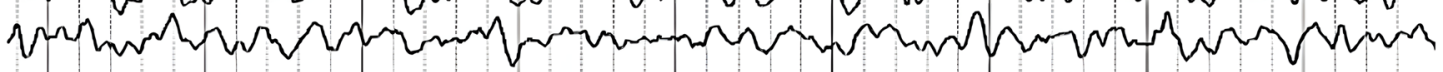

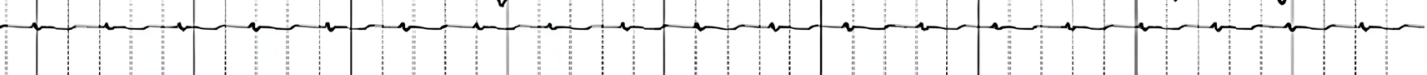

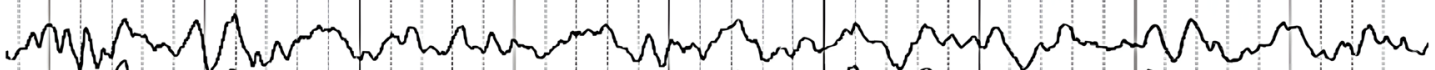

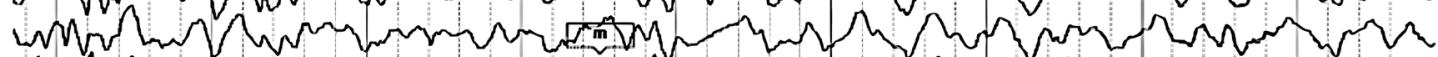

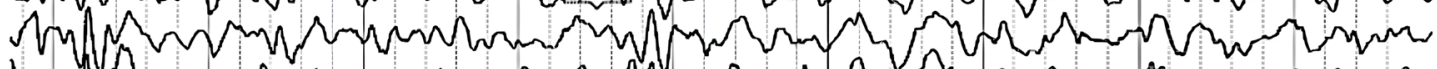

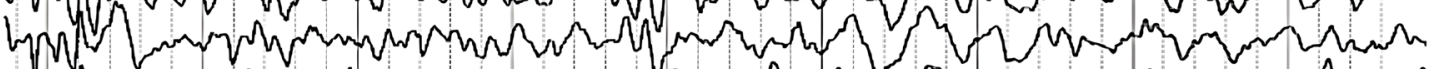

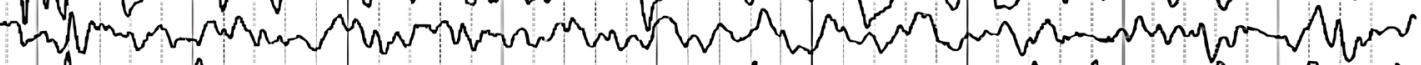

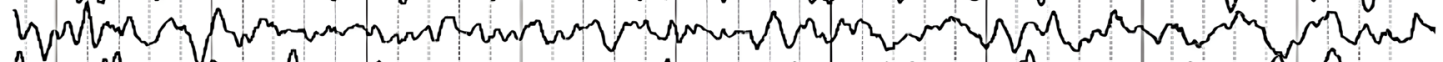

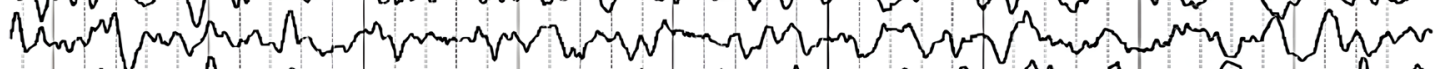

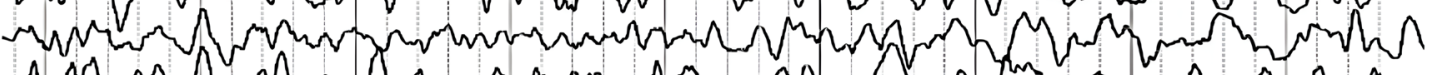

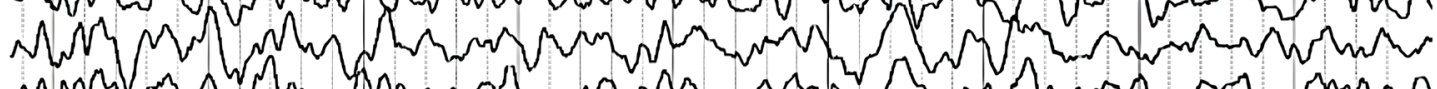
WOV

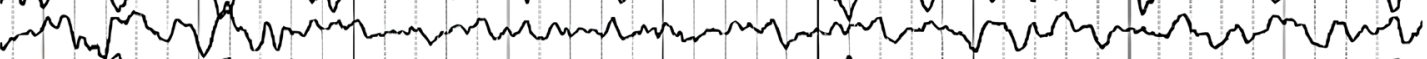

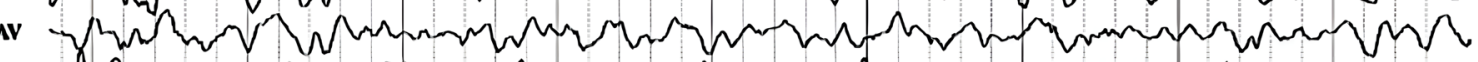

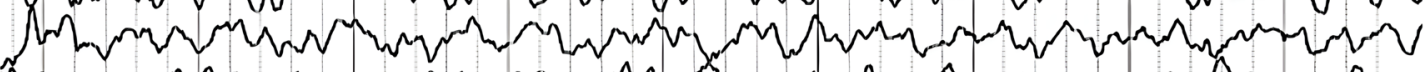

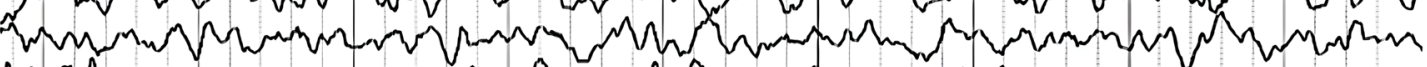

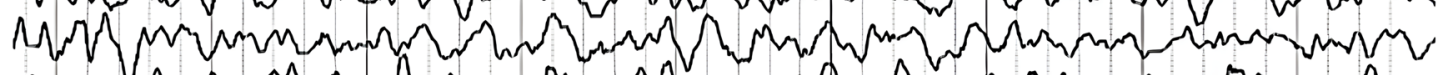

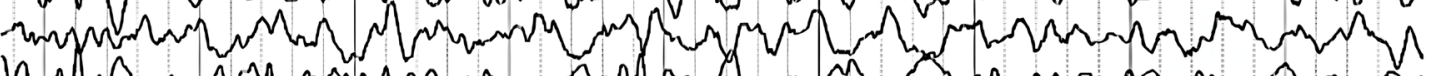
Vol

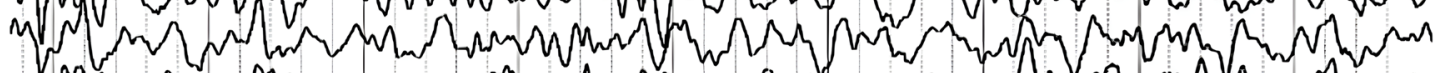

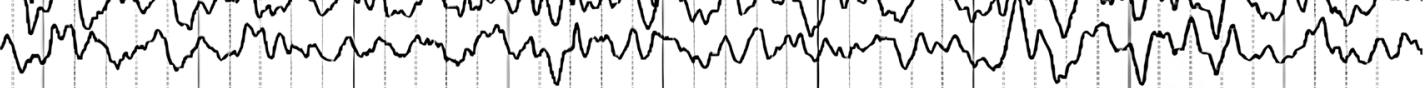

Fig. 2 EEG at age 10 months (on October 22,2019). After 2 months of TPM treatment, the atypical sharp waves were occasionally seen in the bilateral frontal and frontal midline regions during sleep 
A

Fpl-AV

Fp2-AV

F3.AV

Fu-Av

C3.AV

C4.Av

P3.AV

PA.AV

OI.AV

O2.AV

F7.AV

FH-AV

T3-AV

TH-AV

T5.AV

T6-AV

Fi-AV

Ca-AV

ProAv

EKG;

$B$

Fpl-AV

Fp2-AV

F3-AV

F4-AV

C3-AV

C4-AV

P3-AV

P4AV

O1-AV

02-AV

F-AV

FY-AV

Tr-AV

T4-AV

TS-AV

TG-AV

Fr-AV

(n)

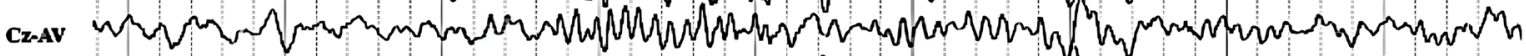
$r_{r} r r_{r} r_{r}$

Slece

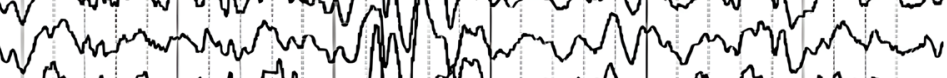

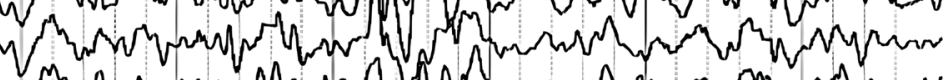

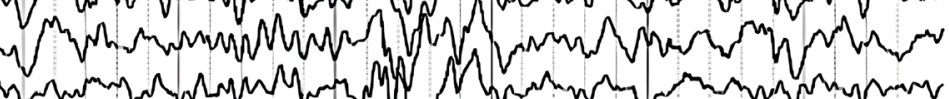

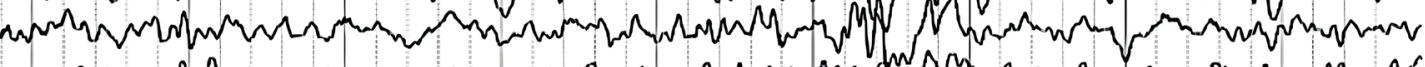

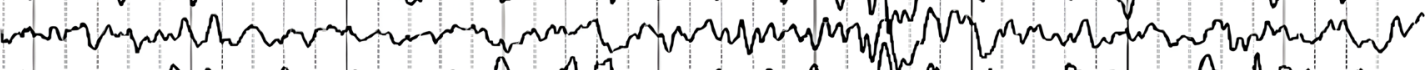

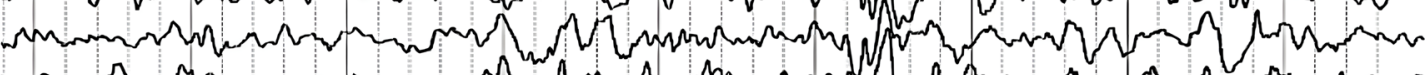

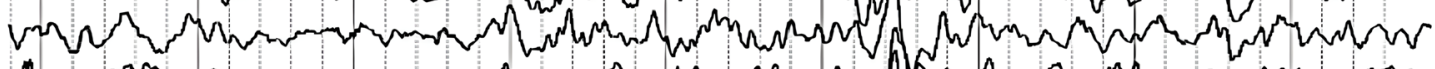

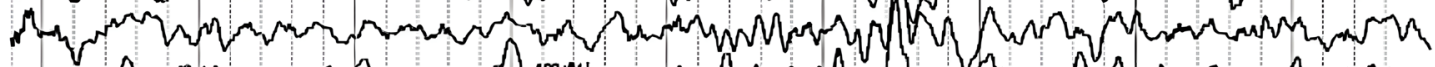

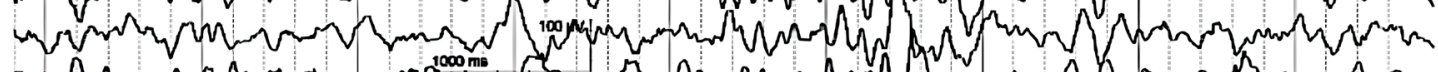

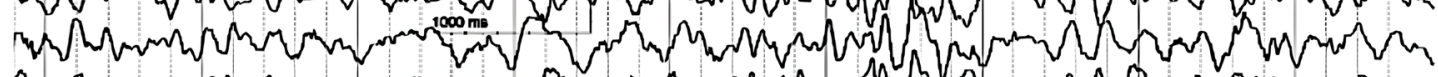

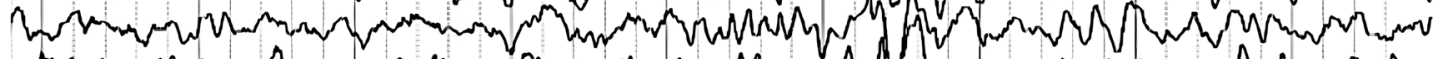

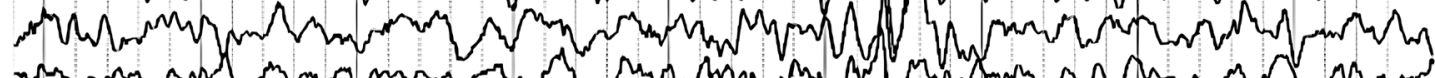

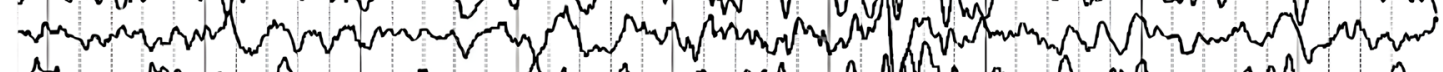

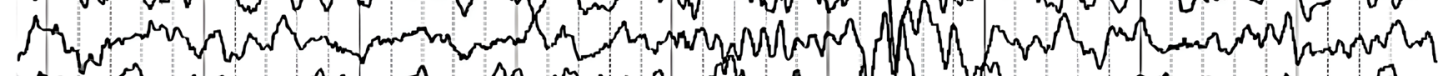

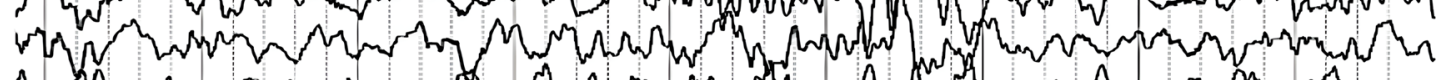

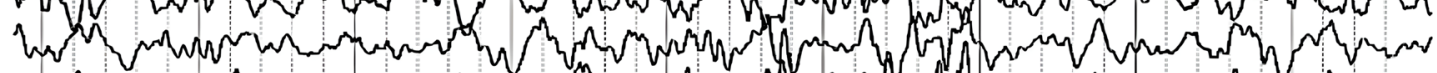

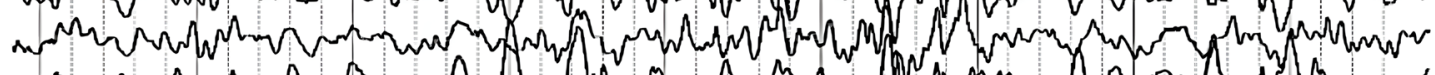

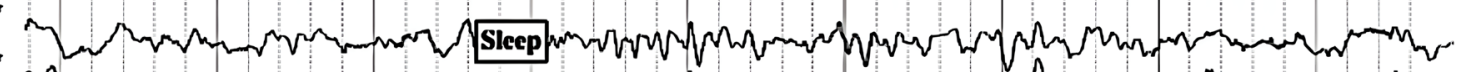

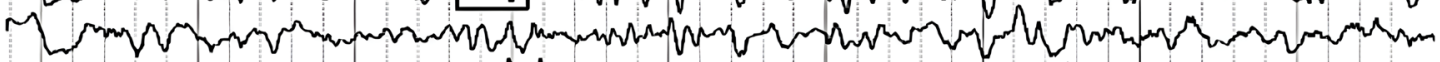

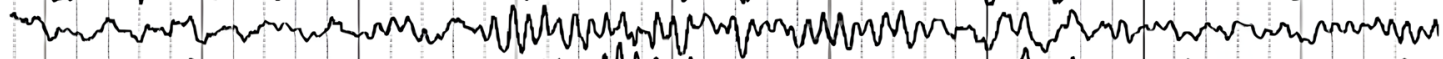

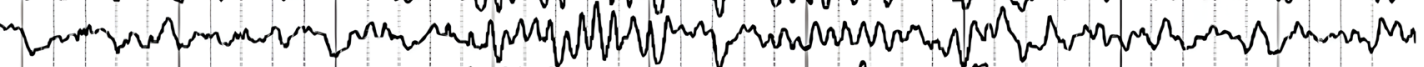

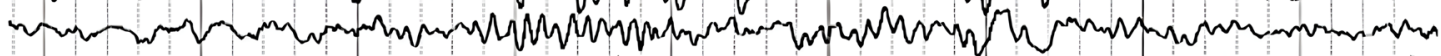

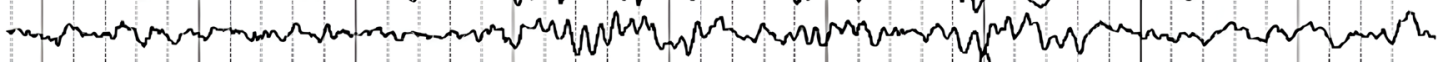

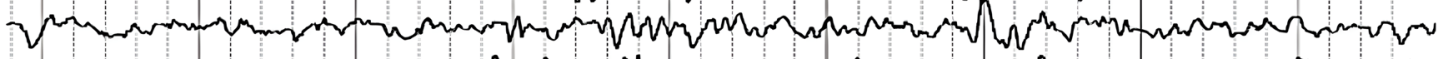

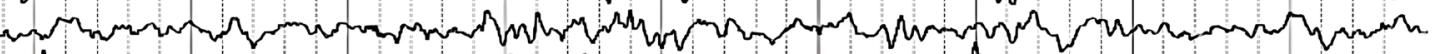

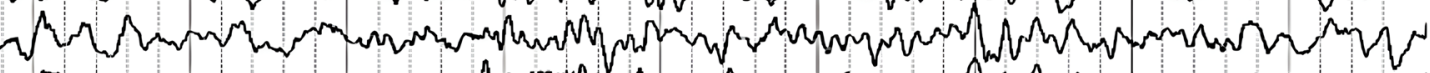

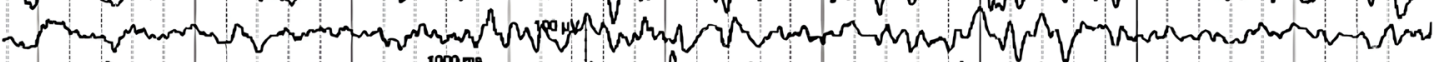

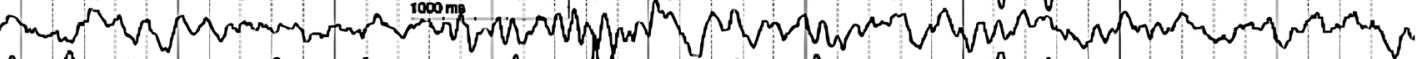

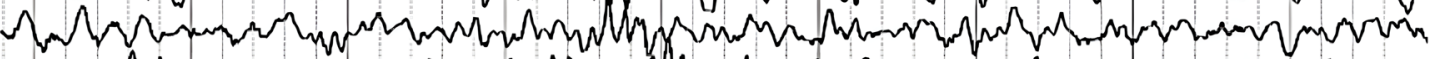

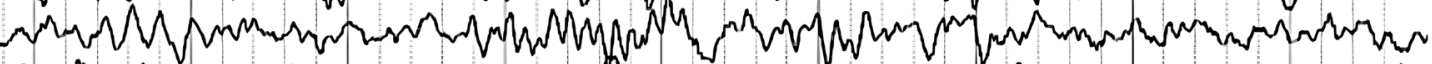

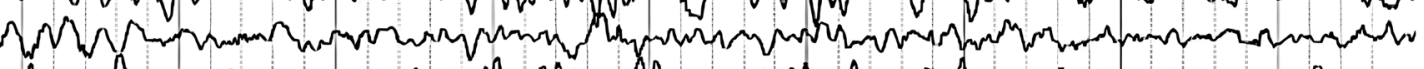

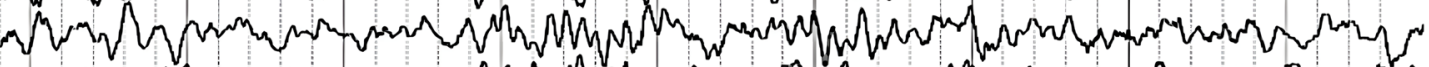

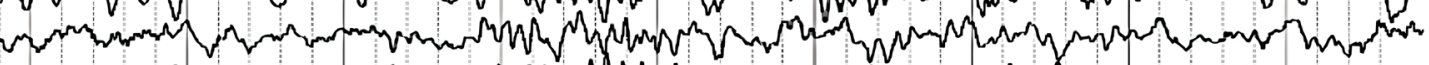

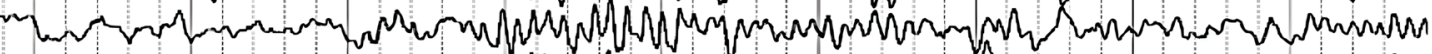

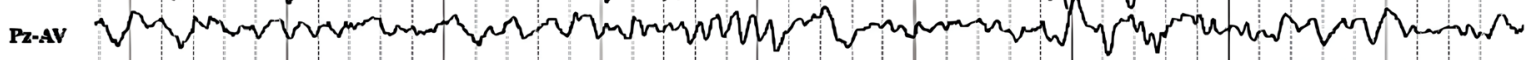
EкG $r-r-p-r+r-r-r-r-r-r+r-r-r$

Fig. 3 EEG at age 1 year and 3 months (on March 19, 2020). After almost 8 months of TPM treatment, EEG showed low-amplitude fast waves when he was awake or sleeping 


\section{The patient}

A A G C T A G G G C G G T T C C G A T C

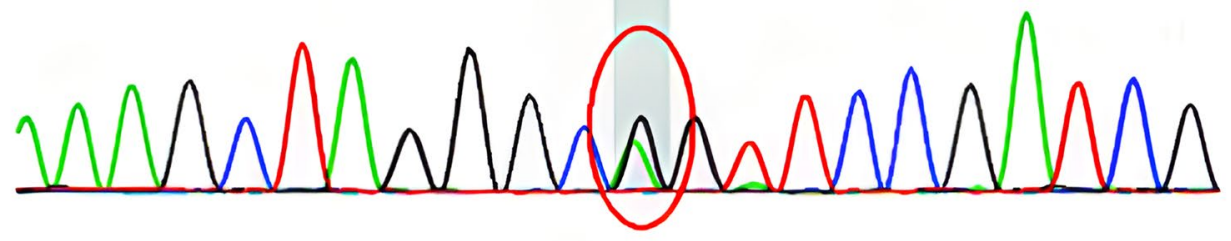

\section{Father}

A A G C T A G G G C G G T T C C G A T C

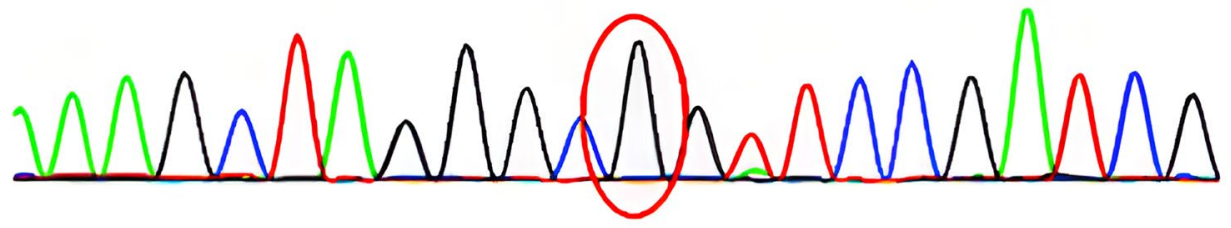

Mother

A A G C T A G G G G G T T C G A T C G

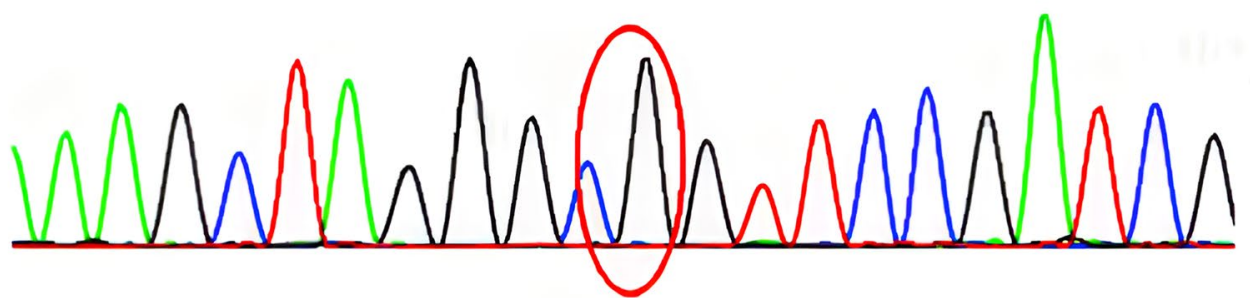

Fig. 4 Genetic analysis results of the child with West syndrome and his parents. A novel heterozygous variant (c.244C > T chr1, 111,147,161,p.R82C) in the KCNA2 gene (NM_004974.3) was detected in the child. No mutation in the KCNA2 gene was detected in his parents 


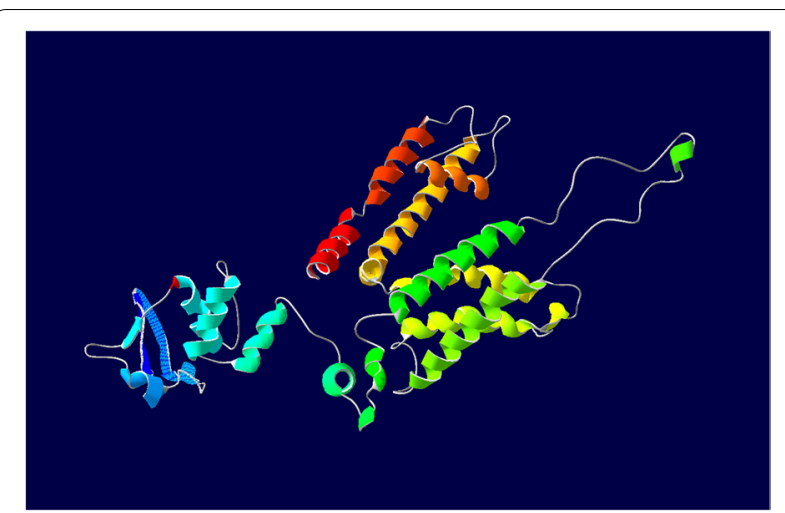

Fig. 5 Protein expression and structure prediction. The mutation occurs in the cytoplasmic $\mathrm{N}$-terminus which is important for tetramerization. Interactions between the different subunits modulate the gating characteristics. The mutation leads to a change in the polarity of the amino acid at the position, which may lead to changes in gating characteristics and ion channel transport efficiency, but the protein function may not be completely lost

axosomatic synapses on Purkinje cells. Kv1.2 plays a key role in the efficient detoxification and synaptic plasticity of neurons, and neuronal membrane repolarization after an action potential, thereby regulating a neuron's electrical excitability [6, 7]. Kv1.2 belongs to the voltage-gated delayed rectifier class of potassium channels, which is composed of four subunits. Different combinations of these subunits lead to various nerve cell types, which can play different roles in different neurons [8-10], and effectively maintain repolarization of the neuronal membrane following an action potential $[7,11]$. Knockout of Kv1.2 gene in mice leads to the development of severe brain stem epilepsy, respiratory failure, excitatory disorder of the CNS, and even premature death at 15 days after birth [12, 13]. Heterozygous deletion of Kv1.2 results in increased seizure susceptibility, and mutations in KCNA2 are also confirmed to be associated with more severe phenotypes. This indicates that the KCNA2 gene mutation interferes with the normal function of Kv1.2 and damages repolarization, leading to hyperexcitability and repetitive discharge tendency in neurons $[7,11,13,14]$.

Several gene functional studies have confirmed that the KCNA2 pathogenic mutations include the loss-offunction (LOF) effect, gain-of-function (GOF) effect, and gain- and loss-of-function effect. These three phenotypes actually overlap with each other to various degrees, but the latter two are more severe [6, 7, 10,14-17]. Mutations causing the LOF effect, the most common phenotype, are almost completely loss-of-function with a dominantnegative effect. This type of patient has seizure onset in infancy or early childhood, and most of them have focal seizures, and there is a trend of multifocal epileptiform discharge in the non-rapid eye movement sleep period, particularly at the back of the head, and the phenomenon of electrical status epilepticus during sleep discharge may appear $[6,10,17]$. Development is normal before disease onset. Intellectual and motor retrogression often appear after onset with mild-to-moderate intellectual disability and ataxia [6]. Mutations causing the GOF effect account for approximately $50 \%$ of $K C N A 2$ encephalopathy, which leads to permanent channel opening, particularly severe epileptic seizures, ataxia and mental retardation, which may be accompanied by hypotonia and myoclonus. Children with GOF mutations and gain- and loss-of-function effect mutations have relatively serious phenotypes and early onset. Epileptic seizures usually occur in the neonatal period, most of which are generalized epilepsy. EEG recording often shows multifocal epileptiform discharges, and the epileptic seizures are severe, usually having no response to many AED therapies. Cerebellar involvement is more common, leading to ataxia in most patients, with shrinking of the cerebellum or even the whole brain. In severe cases, children cannot walk independently, and the prognosis is poor. Most children have severe mental retardation $[6,18,19]$.

Concerning drug treatment, at present, acetazolamide, a carbonic anhydrase, proves to be effective in the treatment of paroxysmal ataxia. In animal experiments, acetazolamide can partially improve motor incoordination in mice with KCNA2 gene mutations. Acetazolamide can rapidly and remarkably improve the epileptic seizures and ataxia of patients with R297Q gene mutation. In addition, a patient with a c.1120A $>\mathrm{G}$ gene mutation shows an apparent quick improvement in the frequency and severity of epileptic seizures after taking acetazolamide $[5,12,15,19]$.

In this report, the case was characterized by generalized seizures that manifest as epileptic spasms and frequent seizures, and diagnosed as early-onset West syndrome. The condition was hard to be controlled with sodium valproate, large doses of vitamin B6 and adrenocorticotropic hormone. EEG recording showed multifocal epileptiform discharges, especially in the bilateral posterior regions, and burst suppression, and interictal EEG showed hypsarrhythmia, similar to the reported GOF effect on EEG discharges. In addition, these abnormal EEG recordings were accompanied by psychomotor retardation and cerebellar involvement without obvious ataxia. This case was therefore considered to be the KCNA2 GOF mutation-related encephalopathy, which usually has a severe phenotype.

TPM is a sulfamate-substituted derivative of the monosaccharide D-fructose $[20,21]$, which may exert its antiseizure effect through five different ways: (1) selectively blocking voltage-gated sodium channels to 
limit the continuously repeated discharges of neurons; (2) slightly modulating the voltage-gated and receptor-gated calcium ion channels [22]; (3) acting on the $\gamma$-aminobutyric acid A (GABA-A) receptor and the GABA transporter 1 to increase the inward chloride ion current caused by GABA, in order to enhance the GABA-mediated inhibitory neurotransmission [5, 23]; (4) inhibiting the release of the excitatory neurotransmitter glutamate and antagonizing ionotropic glutamate receptors, such as a-amino-3-hydroxy-5-methyl-4-isoxazole-propionate receptors to block excitatory neurotransmission mediated by glutamate [23, 24]; and (5) slightly inhibiting the carbonic anhydrase activity $[22,25]$. It has been reported that both sodium valproate and TPM have GABA inhibitory effects, but in this case sodium valproate failed to control epilepsy after 2 months of administration at the beginning of illness, while TPM completely controlled the epileptic seizures after administration for a short time. Therefore, whether the effect of TPM was due to the GABA inhibitory mechanism needs to be further investigated. We believe that TPM as a carbonic anhydrase, can provide slight inhibition of carbonic anhydrase activity, and is effective in treating epileptic seizures in children with KCNA2 mutations [5, 12, 15].

\section{Conclusions}

Although most West syndromes have a poor prognosis, advances in molecular biotechnology and its clinical application have enabled precise finding of etiology in most patients. Etiological diagnosis is becoming more and more important for early clinical intervention and guiding individualized therapy. Many patients can benefit from the etiology-based precise treatment to receive better therapeutic effects. The first report of the case of West syndrome caused by a KCNA2 gene variant advance our understanding of the genetic and phenotypic spectrum of KCNA2-associated disorders. Additionally, we report that TPM treatment is effective, thus providing a clinical reference. However, the exact mechanism underlying the therapeutic effect is still unclear and requires further investigations.

\section{Abbreviations \\ CNS: Central nervous system; EEG: Electroencephalography; GABA-A: Y-aminobutyric acid A; GAT1: GABA transporter 1; GOF: Gain-of-function; LOF: Loss-of-function; TPM: Topiramate; WES: Whole-exome sequencing.}

\section{Acknowledgments}

We thank the patient's parents for providing medical history.

\section{Authors' contributions}

Han Wang and Yanwei Zhu reviewed the literature and wrote the first draft of the manuscript. Hong Chen, Xin Ding and Qi Zeng collected the patient's data and contributed to the first draft. Dezhi Cao was responsible for revision of the manuscript for important intellectual content. Huafang Zou and Jianxiang Liao interpreted the data and wrote a revised version of the manuscript together with Han Wang and Dezhi Cao. All authors contributed to the article and approved the submitted version.

\section{Funding}

This study was sponsored by the Sanming Project of Medicine in Shenzhen (SZSM 201812005), Shenzhen Key Medical Discipline Construction Fund (SZXK033), Epilepsy Research Fund of China Association Against Epilepsy (CUC-2021-02), and Support Project for Clinical Research of Young and Middleaged Doctors in South of the Ten Ridges Neurology (Z20210308).

\section{Availability of data and materials}

The raw data supporting the conclusions of this article are available from the authors, without undue reservation.

\section{Declarations}

\section{Ethics approval and consent to participate}

This research was approved by the Ethics Committee of Shenzhen Children's Hospital and accorded with the Declaration of Helsinki (Register number: 2021014). Informed consent for clinical and genetic analyses was obtained from the parents prior to the study.

\section{Consent for publication}

The informed consent about publication was obtained from the patient's parents.

\section{Competing interests}

The authors have no conflict of interest to declare. The patient gave his informed consent for us to include his details in this report.

Received: 31 July 2021 Accepted: 25 November 2021

Published online: 02 March 2022

\section{References}

1. Wheless JW, Gibson PA, Rosbeck KL, Hardin M, O'Dell C, Whittemore V, et al. Infantile spasms (west syndrome): update and resources for pediatricians and providers to share with parents. BMC Pediatr. 2012;12(1):1-9.

2. Hrachovy RA, Frost JD Jr. Infantile epileptic encephalopathy with hypsarrhythmia (infantile spasms/west syndrome). J Clin Neurophysiol. 2003;20(6):408-25.

3. Wirrell EC, Shellhaas RA, Joshi C, Keator C, Kumar S, Mitchell WG, et al. How should children with west syndrome be efficiently and accurately investigated? Results from the National Infantile Spasms Consortium. Epilepsia. 2015;56(4):617-25.

4. Richards S, Aziz N, Bale S, Bick D, Das S, Gastier-Foster J, et al. Standards and guidelines for the interpretation of sequence variants: a joint consensus recommendation of the American College of Medical Genetics and Genomics and the Association for Molecular Pathology. Genetics Med. 2015;17(5):405-23.

5. Xie G, Harrison J, Clapcote SJ, Huang Y, Zhang JY, Wang LY, et al. A new Kv1.2 channelopathy underlying cerebellar ataxia. J Biol Chem. 2010;285(42):32160-73.

6. Masnada S, Hedrich UBS, Gardella E, Schubert J, Kaiwar C, Klee EW, et al. Clinical spectrum and genotype-phenotype associations of KCNA2related encephalopathies. Brain. 2017;140(9):2337-54.

7. Syrbe S, Hedrich UBS, Riesch E, Djemie T, Muller S, Moller RS, et al. De novo loss- or gain-of-function mutations in KCNA2 cause epileptic encephalopathy. Nat Genet. 2015;47(4):393-9.

8. Christie M, North R, Osborne P, Douglass J, Adelman J. Heteropolymeric potassium channels expressed in Xenopus oocytes from cloned subunits. Neuron. 1990;4(3):405-11.

9. Sheng $M$, Tsaur $M-L$, Jan $Y N$, Jan LY. Contrasting subcellular localization of the Kv1. $2 \mathrm{~K}+$ channel subunit in different neurons of rat brain. J Neurosci. 1994;14(4):2408-17. 
10. Gong P, Xue J, Jiao XR, Zhang YH, Yang ZX. Genotype and phenotype of children with KCNA2 gene related developmental and epileptic encephalopathy. Zhonghua Er Ke Za Zhi. 2020;58(1):35-40.

11. Kearney JA. KCNA2-related epileptic encephalopathy. Pediatric Neurol Briefs. 2015;29(4):27.

12. Brew HM, Gittelman JX, Silverstein RS, Hanks TD, Demas VP, Robinson $\mathrm{LC}$, et al. Seizures and reduced life span in mice lacking the potassium channel subunit Kv1.2, but hypoexcitability and enlarged Kv1 currents in auditory neurons. J Neurophysiol. 2007;98(3):1501-25.

13. Robbins CA, Tempel BL. Kv1.1 and Kv1.2: similar channels, different seizure models. Epilepsia. 2012;53(Suppl 1):134-41.

14. Pena S, Coimbra R. Ataxia and myoclonic epilepsy due to a heterozygous new mutation in KCNA2: proposal for a new channelopathy. Clin Genet. 2015;87(2):e1-3.

15. Hundallah K, Alenizi A, AlHashem A, Tabarki B. Severe early-onset epileptic encephalopathy due to mutations in the KCNA2 gene: expansion of the genotypic and phenotypic spectrum. Eur J Paediatr Neurol. 2016;20(4):657-60.

16. Corbett MA, Bellows ST, Li M, Carroll R, Micallef S, Carvill GL, et al. Dominant KCNA2 mutation causes episodic ataxia and pharmacoresponsive epilepsy. Neurology. 2016;87(19):1975-84.

17. Gong P, Jiao X, Zhang Y, Yang Z. Generation of a human iPSC line from an epileptic encephalopathy patient with electrical status epilepticus during sleep carrying KCNA2 (p. P405L) mutation. Stem Cell Res. 2020;49:102080.

18. Canafoglia L, Castellotti B, Ragona F, Freri E, Granata T, Chiapparini L, et al. Progressive myoclonus epilepsy caused by a gain-of-function KCNA2 mutation. Seizure Eur J Epilepsy. 2019;65:106-8.

19. Lubbers WJ, Brunt E, Scheffer H, Litt M, Stulp R, Browne D, et al. Hereditary myokymia and paroxysmal ataxia linked to chromosome 12 is responsive to acetazolamide. J Neurol Neurosurg Psychiatry. 1995;59(4):400-5.

20. Privitera MD. Topiramate: a new antiepileptic drug. Ann Pharmacother. 1997;31(10):1164-73.

21. Shank RP, Gardocki JF, Vaught JL, Davis CB, Schupsky JJ, Raffa RB, et al. Topiramate: preclinical evaluation of a structurally novel anticonvulsant. Epilepsia. 1994;35(2):450-60.

22. Lhatoo S, Wong I, Sander J. Prognostic factors affecting long-term retention of topiramate in patients with chronic epilepsy. Epilepsia. 2000;41(3):338-41.

23. Rogawski MA, Loscher W, Rho JM. Mechanisms of action of Antiseizure drugs and the ketogenic diet. Cold Spring Harb Perspect Med. 2016;6(5):a022780.

24. Macdonald RL, Kelly KM. Antiepileptic drug mechanisms of action. Epilepsia. 1995;36:S2-S12.

25. Shank R. Investigation of the mechanism of topiramate's anticonvulsant activity. Epilepsia. 1991;32(3):7-8.

Ready to submit your research? Choose BMC and benefit from:

- fast, convenient online submission

- thorough peer review by experienced researchers in your field

- rapid publication on acceptance

- support for research data, including large and complex data types

- gold Open Access which fosters wider collaboration and increased citations

- maximum visibility for your research: over 100M website views per year

At BMC, research is always in progress.

Learn more biomedcentral.com/submissions 